\title{
The IMMUNE-ASSOCIATED NUCLEOTIDE-BINDING 9 Protein Is a Regulator of Basal Immunity in Arabidopsis thaliana
}

\author{
Yuanzheng Wang, ${ }^{1,2}$ Yansha Li, ${ }^{1}$ Tabata Rosas-Diaz, ${ }^{1}$ Carlos Caceres-Moreno, ${ }^{1,2}$ Rosa Lozano-Duran, ${ }^{1}$ \\ and Alberto P. Macho', ${ }^{1,}$ \\ ${ }^{1}$ Shanghai Center for Plant Stress Biology, CAS Center for Excellence in Molecular Plant Sciences; Shanghai Institutes of \\ Biological Sciences, Chinese Academy of Sciences, Shanghai 201602, China; and ${ }^{2}$ University of Chinese Academy of \\ Sciences, Beijing, China
}

Accepted 27 June 2018.

\begin{abstract}
A robust regulation of plant immune responses requires a multitude of positive and negative regulators that act in concert. The immune-associated nucleotide-binding (IAN) gene family members are associated with immunity in different organisms, although no characterization of their function has been carried out to date in plants. In this work, we analyzed the expression patterns of IAN genes and found that IAN9 is repressed upon pathogen infection or treatment with immune elicitors. IAN9 encodes a plasma membrane-localized protein that genetically behaves as a negative regulator of immunity. A novel ian9 mutant generated by CRISPR/Cas9 shows increased resistance to Pseudomonas syringae, while transgenic plants overexpressing IAN9 show a slight increase in susceptibility. In vivo immunoprecipitation of IAN9-green fluorescent protein followed by mass spectrometry analysis revealed that IAN9 associates with a previously uncharacterized C3HC4-type RING-finger domain-containing protein that we named IAN9-associated protein 1 (IAP1), which also acts as a negative regulator of basal immunity. Interestingly, neither ian9 or iap1 mutant plants show any obvious developmental phenotype, suggesting that they display enhanced inducible immunity rather than constitutive immune responses. Because both IAN9 and IAPI have orthologs in important crop species, they could be suitable targets to generate plants more resistant to diseases caused by bacterial pathogens without yield penalty.
\end{abstract}

Current address for Y. Wang: Bio-Agriculture Institute of Shaanxi, Shaanxi Academy of Sciences, Xi' an 710032, China.

${ }^{\dagger}$ Corresponding author: Alberto P. Macho; E-mail: alberto.macho@ sibs.ac.cn

Funding: This work was supported by the Shanghai Center for Plant Stress Biology (Chinese Academy of Sciences). Research in the Macho laboratory is also supported by the National Natural Science Foundation of China (NSFC) (grant 31571973) and the Chinese 1000 Talents Program. Research in the Lozano-Durán laboratory is also supported by the NSFC (grant 31671994) and the 100 Talents Program from the Chinese Academy of Sciences. C. Caceres-Moreno is sponsored by a CAS-TWAS President's Fellowship for International Ph.D. Students. T. Rosa-Diaz is the recipient of a President's International Fellowship Initiative (PIFI) postdoctoral fellowship (number 2016PB042) from the Chinese Academy of Sciences.

*The $\boldsymbol{e}$-Xtra logo stands for "electronic extra" and indicates that 21 supplementary figures and three supplementary tables are published online.

() 2019 The American Phytopathological Society
The plant immune system comprises an intricate network of receptors and regulators aimed at keeping the cellular homeostasis in the absence of pathogen threat and responding rapidly to biotic stimuli in order to prevent infection. Plants have evolved to perceive pathogen-derived molecules that constitute signals of a potential invasion, also called invasion patterns (Cook et al. 2015). Conserved microbial molecules constitute good targets for recognition by plants; some of these molecules have been shown to be perceived by plant cells as pathogenassociated molecular patterns (PAMP) (Boller and Felix 2009). PAMP are perceived at the cell surface by transmembrane pattern-recognition receptors (PRR) (Zipfel 2014). PRR act in coordination with several regulators and additional proteins that mediate signal transduction (Couto and Zipfel 2016), including mitogen-activated protein kinases (MAPK), calcium-dependent protein kinases, receptor-like cytoplasmic kinases, and respiratory burst oxidase homologs (Bigeard et al. 2015; Macho and Zipfel 2014). Downstream responses include the production of the phytohormone salicylic acid (SA) and antimicrobial compounds, the deposition of callose at the cell wall, and extensive transcriptional reprogramming (Boller and Felix 2009). The activation of immunity is aimed at preventing the proliferation of the perceived pathogen, and prepares plant cells to mount an efficient defense response against subsequent biotic threats. However, defense is costly, in terms of energy investment and the concomitant disruption to the normal developmental program (Huot et al. 2014; Stael et al. 2015), and, as such, needs to be tightly regulated. For this reason, immune responses are inducible, and negative regulators ensure a firm control of their activated state. On the other hand, it has been demonstrated that activation of defense and inhibition of growth can be uncoupled, so that active defense and growth can occur simultaneously, indicating that developmental alterations are the consequence of an active process and not necessarily of limiting resources (Campos et al. 2016; de Wit et al. 2013; Scheres and van der Putten 2017).

Pathogens have developed strategies to manipulate plant cells in order to proliferate inside plant tissues. These include the suppression of plant immunity, the alteration of the physical environment, and the acquisition of nutrients to support their pathogenic lifestyle (Win et al. 2012). In gram-negative bacterial pathogens, the most important virulence factor is the type-III secretion system, which injects effector proteins directly into plant cells (type-III-secreted effectors [T3E]). The manipulation of plant cellular functions by T3E is essential for bacteria to proliferate and is required for the development of 
disease (Macho 2016; Macho and Zipfel 2015). However, some plants have evolved intracellular receptors that can perceive $\mathrm{T} 3 \mathrm{E}$ activities as an indication of pathogen invasion, hence becoming resistant to bacterial infection. These receptors contain nucleotide-binding and leucine-rich repeat domains (NLR) (Khan et al. 2016). NLR activation contributes to the development of defense responses similar to those established after PRR activation but are often more intense and sometimes lead to local cell death, which prevents further pathogen proliferation and spread (Chiang and Coaker 2015). Both PRR and NLR constitute the basis of plant innate immunity, and are the major determinants of basal immunity in plants.

Guanosine triphosphate (GTP)-binding proteins are regulators of various biological processes in eukaryotic cells such as signal transduction, cell proliferation, cytoskeletal organization, and intracellular membrane trafficking, and are classified into numerous families (Takai et al. 2001; Vernoud et al. 2003). IMMUNE-ASSOCIATED NUCLEOTIDE-BINDING/GTPases OF IMMUNITY-ASSOCIATED PROTEINS (IAN/GIMAP) comprise a subfamily of GTPase-like proteins that has been found in anthozoans, vertebrates, and angiosperm plants (Nitta and Takahama 2007; Weiss et al. 2013). In vertebrates, proteins from the IAN/GIMAP family regulate the development and homeostasis of $\mathrm{T}$ cells and are associated with autoimmunity (Nitta and Takahama 2007). The transcriptional regulation of genes encoding IAN/GIMAP has been linked to immunity in different organisms: in mice, IAN/GIMAP genes are mostly
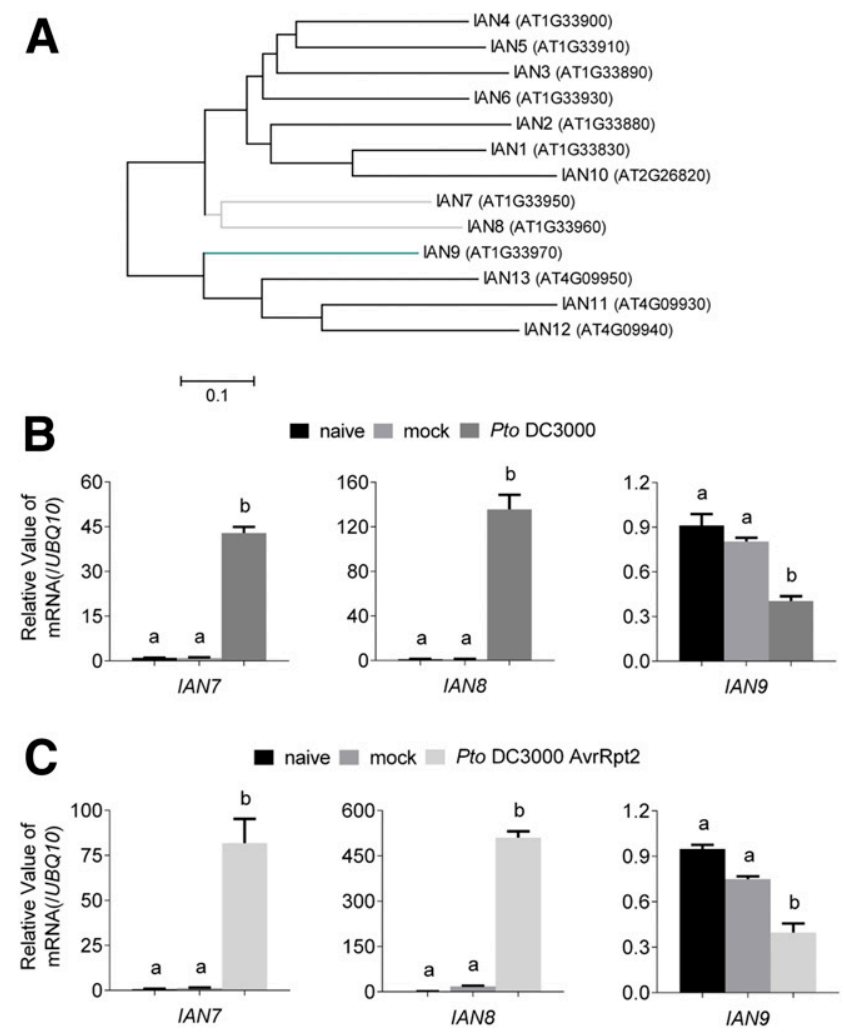

Fig. 1. Transcription patterns of the immune-associated nucleotide-binding (IAN) gene family in Arabidopsis. A, Phylogenetic tree of IAN proteins. The scale bar denotes a relative measure of evolutionary distance. $\mathbf{B}$ and $\mathbf{C}$, Relative expression of IAN7, IAN8, and IAN9 in 9- to 10-day-old Arabidopsis seedlings, $6 \mathrm{~h}$ after inoculation with Pseudomonas syringae pv. tomato DC3000 (B) or DC3000 (AvrRpt2) (C). Real-time quantitative polymerase chain reaction results were normalized with UBIQUITIN 10 (UBQ10, AT4G05320). Values are means \pm standard error of the mean $(n=$ 3 biological replicates; see Materials and Methods). Statistical differences were calculated using one-way analysis of variance $(P<0.01)$. Each experiment was performed three times with similar results. expressed in immune tissues (Nitta et al. 2006), and they have also been reported as induced in corals after treatment with the bacterial immune elicitor muramyl dipeptide (Weiss et al. 2013). In Arabidopsis, IAN/GIMAP are defined by the presence of an avrRpt2-induced gene 1 (AIG1) domain, and contain conserved GTP-binding domains, including a P-loop motif known to bind GTP/GDP in Ras GTPases (Bourne et al. 1991) and coiled-coil motifs (Liu et al. 2008). Originally, AtAIG1 (also known as IAN8) was defined as a gene overexpressed in response to the avirulent bacterial strain Pseudomonas syringae pv. maculicola expressing the effector AvrRpt2 (Reuber and Ausubel 1996). Additionally, computational analysis of transcriptomic data has revealed that the transcription of other IAN family members responds to different biotic stimuli: nematode infection induces the expression of IAN3 and IAN11, while the transcription of IAN11, IAN12, and IAN13 is reduced by infection with Myzus persicae (Liu et al. 2008).

Despite the accumulating evidence that associates IAN genes with immunity in plants, no characterization of their function in this process has been carried out to date. Here, we analyzed the expression patterns of $I A N$ genes and found that the expression of IAN9 is repressed upon pathogen infection or treatment with immune elicitors. Further characterization indicated that IAN9 encodes a plasma membrane (PM)-localized protein and that it genetically behaves as a negative regulator of immunity. In vivo immunoprecipitation (IP) of IAN9-green fluorescent protein (GFP) followed by mass spectrometry (MS) analysis revealed that IAN9 associates with a C3HC4-type RING-finger domaincontaining protein that we named IAN9-associated protein 1 (IAP1). Interestingly, our results show that IAP1, like IAN9, negatively regulates immunity, raising the idea that these two proteins may work together in the control of immune responses.

\section{RESULTS}

\section{Expression analysis of the $I A N$ gene family upon bacterial infection reveals differential expression patterns for IAN7, IAN8, and IAN9.}

The IAN protein family in Arabidopsis is composed of 13 members (Fig. 1A) (Liu et al. 2008). Phylogenetic analysis of IAN amino acid sequences shows a clear separation into different subgroups (Fig. 1A; Supplementary Fig. S1). Previous reports have described changes in expression of IAN gene family members upon different biotic stimuli (Liu et al. 2008; Reuber and Ausubel 1996). In order to characterize the transcriptional response of $I A N$ genes to bacterial infection, we inoculated 9- and 10-day-old Arabidopsis seedlings with the virulent pathogen $P$. syringae pv. tomato DC3000. Three IAN genes showed differential expression patterns upon bacterial infection: IAN7 and IAN8 showed a significant upregulation, while IAN9 showed a significant downregulation (Fig. 1B). We did not detect mRNA for IAN1, -2, -4, -6, -10, -11, -12, or -13 , suggesting that these genes are not expressed in 10-day-old Arabidopsis seedlings in our experimental conditions. The pathogen-induced upregulation of IAN8 is reminiscent of the originally reported upregulation of this gene by bacteria expressing the effector AvrRpt2, which induces activation of the plant NLR RPS2 (Reuber and Ausubel 1996). Accordingly, we found that the differential expression of IAN7 or -8 and IAN9 also takes place upon infection of Arabidopsis rosette leaves with $P$. syringae pv. tomato expressing AvrRpt2 (Fig. 1C). The particular expression pattern of IAN9 among the IAN gene family suggests an exclusive function for IAN9 rather than functional redundancy with other IAN family members. This idea is supported by the fact that IAN9 constitutes a specific phylogenetic group within the IAN gene family (Fig. 1A). For these reasons, we decided to focus our attention on IAN9. 
IAN9 expression is reduced upon chemical activation of plant immunity.

IAN9 is broadly expressed in cotyledons, hypocotyls, and roots of Arabidopsis seedlings (Supplementary Fig. S2). To dissect the bacteria-induced repression of IAN9 transcription, we sought to determine whether perception of purified immune elicitors affects IAN9 expression. For this purpose, we first treated Arabidopsis seedlings with the flagellin-derived peptide flg22, which is widely used as an elicitor of immune responses. Our results show that IAN9 expression is significantly reduced $1 \mathrm{~h}$ after flg22 treatment (Fig. 2A). The perception of different invasion patterns, including flg22, leads to the production of the phytohormone SA, which is a key player in the activation of plant immunity against biotrophic pathogens (Vlot et al. 2009). Treatment with SA for $3 \mathrm{~h}$ led to a reduction of IAN9 expression, although the abundance of IAN9 transcripts returned to basal levels after a 6-h SA treatment (Fig. 2B), revealing a transient downregulation of IAN9 transcript abundance upon SA treatment. Finally, to determine whether the reduction on IAN9 transcription upon flg22 treatment depends on the downstream SA accumulation (Tsuda et al. 2008), we performed flg22 treatment in the SA-depleted sid2/NahG plants, deficient in pathogen-induced SA biosynthesis (Wildermuth et al. 2001) and constitutively expressing the bacterial salicylate hydroxylase NahG, which degrades SA (Delaney et al. 1994). Interestingly, the flg22-triggered downregulation of IAN9 transcript abundance was also observed in sid2/NahG plants (Fig. 2C), suggesting that SA is not required for the flg22-induced transcriptional repression of IAN9.

IAN9 localizes to the PM through its $\mathrm{C}$ terminus.

In order to investigate the subcellular localization of IAN9, we generated stable transgenic Arabidopsis lines expressing an N-terminal GFP-tagged IAN9 protein (see below for a detailed characterization of these lines), and used confocal microscopy to determine the localization of GFP-IAN9. Contrary to free GFP, which shows a nuclear/cytoplasmic localization, GFPIAN9 specifically localized at the cell periphery (Fig. 3A). This localization was similar to that observed for well-characterized PM-localized proteins, such as the brassinosteroid (BR) receptor BRI1 (Wang et al. 2001) (Fig. 3A). To determine whether GFP-IAN9 is associated with membranes, we used the lipophilic fluorescent dye FM4-64, which is rapidly incorporated into membranes upon contact with plant cells (Bolte et al. 2004; Fischer-Parton et al. 2000). Our results show that GFP-IAN9 fluorescence colocalizes with FM4-64-labeled compartments (Fig. 3B and C), similar to BRI1-GFP fluorescence and different from free GFP (Fig. 3B and C). To further confirm that IAN9 localizes at the PM, we performed plasmolysis assays by treating plant tissues with $1 \mathrm{M} \mathrm{NaCl}$. Upon plasmolysis, both GFP-IAN9 and BRI1-GFP were detected in Hechtian strands, which represent sites of incomplete PM retraction from the cell wall (Fig. 3D). Altogether, our microscopy analysis indicates that IAN9 localizes at the PM in plant cells.

The C-terminal domain is not conserved among IAN proteins. Interestingly, the C-terminal sequence of IAN9 shows an overrepresentation of positively charged amino acids (KKLRENLERAEKETKELQKKLGKCINL; $33.3 \%$ of R/K), not present in other IAN proteins (Supplementary Fig. S3), which could mediate an interaction with the negatively charged phospholipids of the PM. To test this hypothesis, we generated Arabidopsis stable transgenic lines expressing a truncated GFPIAN9 version lacking the $27 \mathrm{C}$-terminal amino acids (IAN9 $\Delta \mathrm{C}$ 27). The IAN9 $\Delta \mathrm{C}-27$ version lost the specific PM localization seen for wild-type GFP-IAN9, and was mostly detected in the cytoplasm. Additionally, we found that, when GFP is fused to the C-terminal end of IAN9 (IAN9-GFP), this protein loses its specific PM localization, and is mostly found in the cytoplasm. Compared with IAN9, the IAN8 C terminus has a lower representation of positively charged amino acids (18.5\%), and we found that an N-terminal GFP-tagged IAN8 (GFP-IAN8) localizes to the cytoplasm. Altogether, these results suggest that the exclusive C-terminal domain of IAN9 is required for its localization at the PM.

Generation of IAN9 knock-out and overexpression lines.

Public repositories for Arabidopsis T-DNA insertion lines contain four independent lines with T-DNA insertions in the IAN9 genomic locus: SAIL_167_B02, SALK_534_B01, $S A L K \_144369$, and $G K-146 B 08$. For all of these lines, the insertion site is located in the predicted promoter region of IAN9. Among them, $G K-146 B 08$ is the line harboring the nearest TDNA insertion to the IAN9 start codon (Supplementary Fig. S4) and, therefore, we chose this line for further analyses. Sequencing analysis results showed that the insertion site is located 76 bp upstream of the 5' untranslated region of the IAN9 gene, and 319 bp upstream of the IAN9 start codon. Reversetranscription polymerase chain reaction (RT-PCR) and quantitative RT-PCR (RT-qPCR) analyses showed that this mutant has approximately a threefold reduction in the amount of IAN9 transcripts, indicating that this line is a knockdown ian9
A

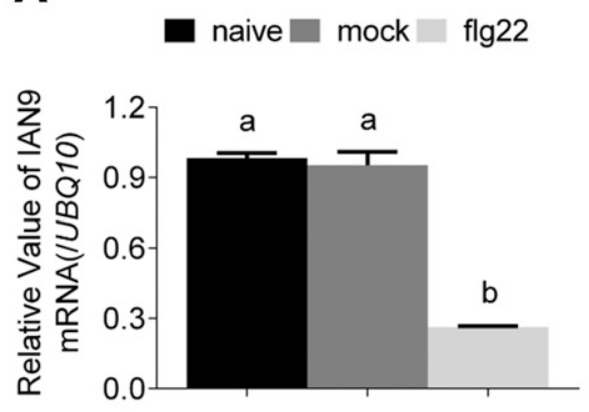

WT
B
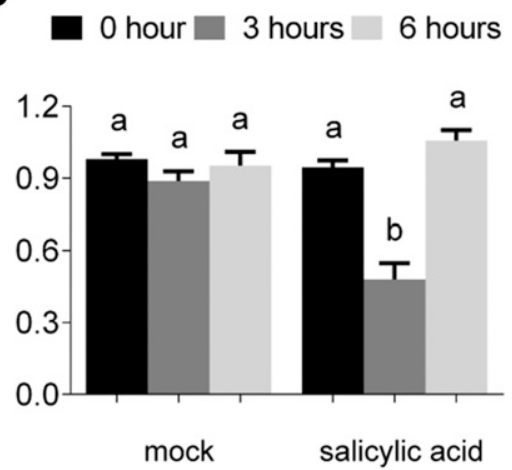

C

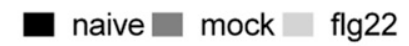

Fig. 2. Treatment with flg22 or salicylic acid causes a reduction on the transcription of immune-associated nucleotide-binding (IAN) 9 . Relative expression of IAN9 in 9- to 10-day-old Arabidopsis seedlings. WT = wild type. A and C, Seedlings were treated with $100 \mathrm{nM}$ flg22 for $1 \mathrm{~h}$. B, Seedlings were treated with $0.5 \mathrm{mM}$ salicylic acid for 3 or $6 \mathrm{~h}$. Real-time quantitative polymerase chain reaction results were normalized with UBIQUITIN 10 (UBQ10, AT4G05320). Values are means \pm standard error of the mean ( $n=3$ biological replicates; see Materials and Methods). Statistical differences were calculated using one-way analysis of variance $(P<0.01)$. Each experiment were performed three times with similar results. 
mutant. To further characterize this line, we determined the IAN9 transcript levels upon bacterial inoculation. Surprisingly, we found that IAN9 transcript levels increased significantly upon inoculation with $P$. syringae pv. tomato or AvrRpt2, reaching higher levels than those observed in wild-type plants (Fig. 1). These findings indicate that the T-DNA insertion in the IAN9 promoter generates an aberrant expression pattern of IAN9 in this line, rendering it unusable for the functional characterization of this gene.

In order to perform genetic analysis of the contribution of IAN9 to plant immunity, we generated ian 9 mutant lines using CRISPR/Cas9-assisted genome editing (Feng et al. 2013; Mao et al. 2013). We selected the best predicted target site in the IAN9 gene sequence for recognition by the Cas9/sgRNA complex (Supplementary Fig. S5), and performed the targeted mutagenesis as explained below (Materials and Methods). Sequencing of the resulting line showed an addition of $1 \mathrm{bp}$ in the second exon of IAN9, creating a premature stop codon $366 \mathrm{bp}$ downstream of the start codon (Supplementary Fig. S6), which generates a truncated protein with a disrupted GTP-binding domain. Upon selection of seedlings containing the ian 9 mutation in homozygosis, we selected a line in which the Cas9 gene was segregated out; this Cas9-free ian9 line was used for further experiments. Additionally, as mentioned before, we generated Arabidopsis transgenic lines overexpressing IAN9 in a Col-0 wild-type background, using a $35 \mathrm{~S}$ promoter to express a GFP-IAN9 fusion. We selected two independent homozygous lines that accumulated detectable amounts of GFP-IAN9 fusion protein, and higher transcript levels of IAN9 compared with those in the Col-0 wild type (overexpression [OE]-GFPIAN9\#3-10 and OE-GFP-IAN9\#7-1). As controls, we selected two independent homozygous lines expressing free GFP, which did not show changes in IAN9 transcription and accumulated detectable levels of free GFP. Interestingly, although the $35 \mathrm{~S}$ promoter led to high IAN9 expression compared with the Col0 wild type, bacterial infection still caused a reduction in IAN9 transcript levels (Supplementary Fig. S7), suggesting a posttranscriptional regulation of the abundance of IAN9 transcripts. Overexpression of IAN9 did not affect IAN8 expression in basal conditions or upon bacterial infection.

\section{Plant growth and early immune responses} are not affected by altered expression of IAN9.

Both IAN9-overexpressing or ian9 knockout seedlings and adult plants were visually indistinguishable from the wild type (Supplementary Figs. S8 and S9). Given the predicted association of the IAN family with immune responses and the alteration in IAN9 expression upon elicitation with flg22, we sought to determine whether IAN9 is involved in early PAMPtriggered immunity (PTI) responses; namely, the flg22triggered burst of reactive oxygen species (ROS) and the activation of a cascade of MAPK (Boller and Felix 2009, Macho and Zipfel 2014). Our results show that mutation or overexpression of IAN9 did not have a detectable impact in the dynamics or total accumulation of ROS upon flg22 treatment (Supplementary Fig. S10). Similarly, neither mutant nor OE lines showed differences in flg22-triggered MAPK activation compared with wild-type plants or free GFP-expressing
A GFP
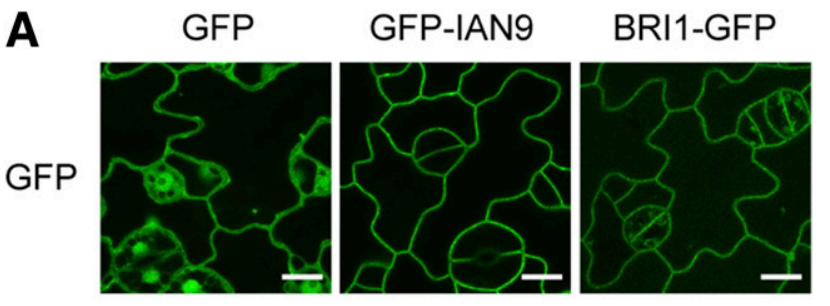

B
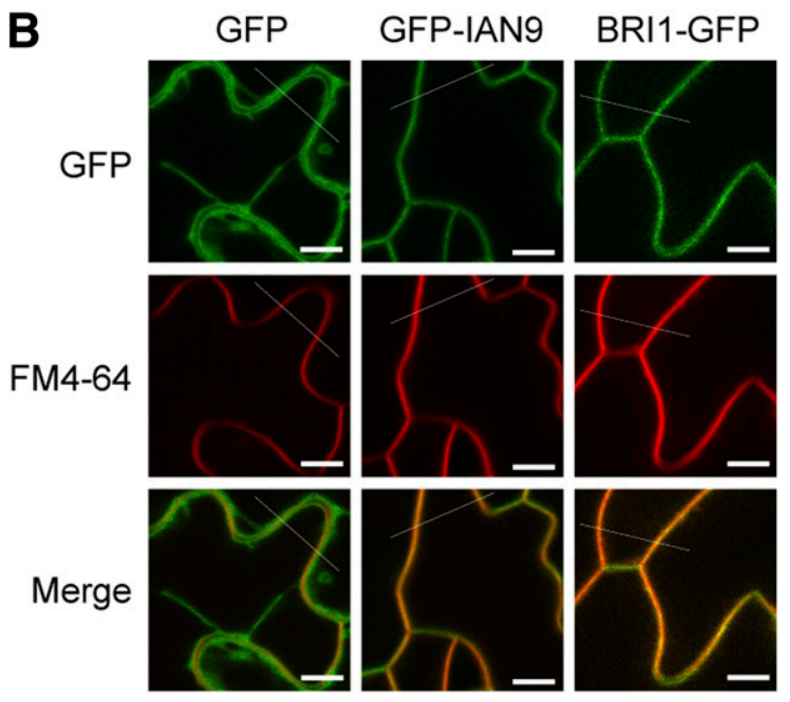
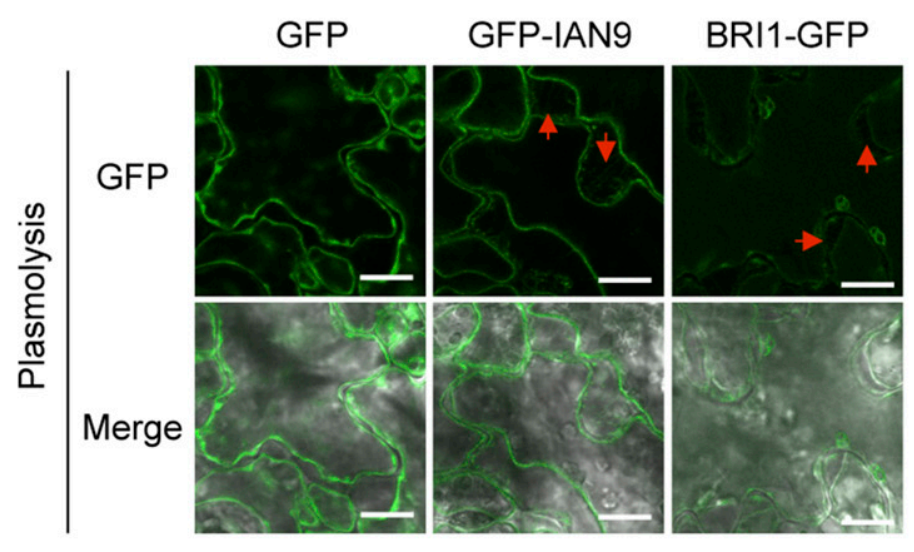

Fig. 3. Green fluorescent protein (GFP) immune-associated nucleotide-binding (IAN)9 localizes to the plasma membrane in Arabidopsis. A, Confocal images of GFP, GFP-IAN9, and BRI1-GFP in Arabidopsis cotyledon epidermal cells. Bar $=10 \mu \mathrm{m}$. B, Confocal images of GFP, GFP-IAN9, and BRI1-GFP in Arabidopsis cotyledon epidermal cells. Arabidopsis seedlings were treated FM4-64 for 5 min before confocal imaging, and the FM4-64 signal is shown in red. Bar $=5 \mu \mathrm{m}$. C, Fluorescence intensity through the thin lines shown in B. D, Confocal images of GFP, GFP-IAN9, and BRI1-GFP in Arabidopsis cotyledon epidermal cells after plasmolysis $(5$-min treatment with $1 \mathrm{M} \mathrm{NaCl})$. Arrows indicate the presence of Hechtian strands. Bar $=10 \mu \mathrm{m}$. 
controls. Taken together, these results suggest that alterations of IAN9 expression do not affect early PTI responses.

\section{IAN9 negatively regulates plant immunity against $P$. syringae pv. tomato $\mathrm{DC} 3000$.}

To test whether IAN9 contributes to plant immunity against bacterial pathogens, we performed surface inoculation of the ian9 mutant line with $P$. syringae pv. tomato DC3000 and determined bacterial replication in plant tissues. Our results show that knockout mutation of IAN9 increased plant resistance against DC3000, causing a 13-fold reduction of bacterial titers (Fig. 4A). This increase in disease resistance does not seem to be caused by differences in basal expression of SA-dependent defense-related genes (Supplementary Fig. S11). In order to test whether the increase in resistance also occurs in a context of effector-triggered immunity, we syringe infiltrated Arabidopsis rosette leaves with DC3000 (AvrRpt2). However, no differences were detected in terms of replication of this strain (Fig. 4B). To confirm that the observed increased resistance to DC3000 is really due to the absence of ian9, we generated complementation lines expressing GFP-IAN9, driven by a $35 \mathrm{~S}$ promoter, in the ian9 knockout mutant background, where the GFP-IAN9 protein accumulated and localized to the PM (Supplementary Fig. S12). Expression of GFP-IAN9 in the ian9 background was able to rescue the level of growth of DC3000 to that observed in wildtype plants, confirming the association of IAN9 with the observed increased resistance. Interestingly, transgenic lines overexpressing GFP-IAN9 in a wild-type background showed a tendency to support higher bacterial loads compared with wildtype or GFP-expressing lines, suggesting that IAN9 overexpression suppresses plant immunity against DC3000, although such a tendency was not always reproducible or statistically significant across eight independent biological repeats (Supplementary Fig. S13). This trend was not observed when plants were inoculated with a hypovirulent DC3000 derivative unable to produce the virulence factor coronatine (DC3000 COR-) (Supplementary Fig. S14) (Melotto et al. 2006).

\section{Identification of IAN9-interacting proteins.}

To characterize further the mode of action of IAN9, we searched for proteins physically associated with IAN9 in plant cells using Arabidopsis seedlings expressing GFP-IAN9 (line OE-GFP-IAN9\#3-10) and seedlings expressing free GFP as control. Upon GFP IP using agarose beads coupled to an antiGFP nanobody (GFP-Trap beads), we detected associated proteins using liquid chromatography coupled to tandem MS (LC-MS/MS). In order to detect potential dynamic interactions, we additionally treated seedlings for $1 \mathrm{~h}$ with flg22 or SA before IP. Two independent biological replicates showed a large number of proteins physically associated with IAN9, which we filtered using the following criteria: (i) presence in both biological replicates, (ii) detection of two or more exclusive unique peptides, and (iii) absence in the GFP control. After applying these filters, 14 proteins were identified as IAN9 candidate interactors (Supplementary Table S1).

Among these candidate interactors, we drew our attention to an uncharacterized protein encoded by the AT1G18660 gene, which we named IAP1. Although our experimental approach does not provide a quantitative assessment of protein interactions, we noticed that the number of detected IAP1 peptides decreased in samples treated with SA, whereas it did not change substantially in samples treated with flg22. Domain analysis predicts the presence of three tetratricopeptide-like helical (TPR) domains, a C3HC4-type RING-finger domain, and an ATP-dependent protease $\mathrm{La}(\mathrm{LON})$ domain in this protein (Supplementary Fig. S15). Upon Agrobacterium-mediated transient expression in Nicotiana benthamiana, a GFP-IAP1 fusion protein localized at the cell periphery, nucleus (weakly), and around the nucleus; the latter most likely corresponds to the usual endoplasmic reticulum localization of PM-localized proteins overexpressed in this system. Coexpression with red fluorescent protein (RFP)-IAN9 showed that both proteins colocalize upon transient expression (Fig. 5A). To confirm the interaction between IAN9 and IAP1, we performed a targeted co-IP using GFP-IAP1 and an N-terminal fusion of IAN9 to the C-terminal domain of luciferase (Cluc-IAN9). Co-IP assays show that GFPIAP1 interacts with Cluc-IAN9 but not with Cluc alone (Fig. 5B); Cluc-IAN9 does not interact with free GFP (Supplementary Fig. S16). To determine whether the IAN9-IAP1 interaction is direct, we performed a luciferase complementation assay, transiently coexpressing Cluc-IAN9 and IAP1 fused to the N-terminal domain of luciferase (IAP1-Nluc). A positive control coexpressing AtSGT1b-Nluc and Cluc-AtRAR1 showed a strong luciferase signal (Supplementary Fig. S17), as described before (Chen et al. 2008). In contrast, tissues coexpressing Cluc-IAN9 and NlucIAP1 did not show any detectable luciferase signal, although both proteins accumulated. As an alternative technique to detect direct interactions, we employed fluorescence resonance energy transfer-fluorescence-lifetime imaging microscopy (FRETFLIM) by coexpressing GFP-IAP1 and RFP-IAN9. However, no difference in lifetime GFP fluorescence was detected when compared with control samples. Although an influence of the tags cannot be ruled out, these results suggest that the interaction observed for IAP1 and IAN9 is most likely indirect.

\section{IAP1 negatively regulates plant immunity against $P$. syringae pv. tomato $\mathrm{DC} 3000$.}

Public repositories for Arabidopsis T-DNA insertion lines contain two independent lines with T-DNA insertions in the IAP1 genomic locus: SALK_119114 and SALK_093498 (Supplementary Fig. S18). We isolated homozygous lines containing these insertions and confirmed the absence of IAPI transcripts, naming these lines iapl-1 and iap 1-2, respectively. Both lines displayed wild-type-like growth and development when grown on soil under short-day conditions (Fig. 6A), although mutant seedlings showed a slightly reduced root length compared with the Col-0 wild type when grown in vertical MS plates (Supplementary Fig. S19).

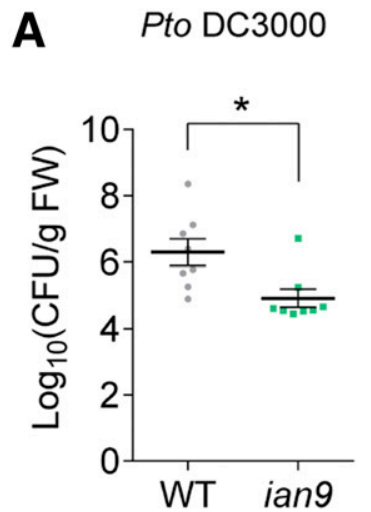

B Pto DC3000 AvrRpt2

Fig. 4. Immune-associated nucleotide-binding (IAN)9 negatively regulates plant immunity against Pseudomonas syringae pv. tomato DC3000. A, Growth of surface (spray)-inoculated DC3000 (optical density at $600 \mathrm{~nm}$ $\left.\left[\mathrm{OD}_{600}\right]=0.1\right)$ in wild-type (WT) Col-0 and ian9 mutant plants, 3 days postinoculation (dpi). Experiments were repeated more than three times with similar results. B, Growth of DC3000 (AvrRpt2) $\left(\mathrm{OD}_{600}=0.001\right)$ infiltrated with a needleless syringe into WT Col-0 and ian 9 mutant plants, 3 dpi. Experiments were performed twice with similar results. Data were represented as means \pm standard error ( $n=8$ independent plants) (A and B). Statistical differences were calculated using a Student's $t$ test; "ns" indicates no significant difference and an asterisk indicates significant difference $(P<0.05)$ 
To determine whether mutations in IAPI have an impact on plant resistance against bacterial pathogens, we performed surface inoculation of the iapl mutant lines with $P$. syringae pv. tomato DC3000. Our results show that both iap1-1 and iap1-2 mutant lines are more resistant than the Col-0 wild type against DC3000, showing a 19-fold reduction in bacterial loads (Fig. 6B). However, none of these lines showed differences after inoculation with $P$. syringae pv. tomato expressing AvrRpt2 (Fig. 6C). This enhancement of disease resistance did not seem to be caused by differences in basal expression of SAdependent defense-related genes. Interestingly, these results resemble those obtained during the characterization of the ian 9 knockout mutant line (Fig. 4), suggesting that both proteins are involved in the negative regulation of basal immunity against bacterial pathogens, and may act in the same pathway through physical association.

\section{DISCUSSION}

In this work, we characterized the transcription patterns of IAN family members in Arabidopsis upon infection with the bacterial pathogen $P$. syringae pv. tomato DC3000. IAN7 and $I A N 8$, which seem to form a separate phylogenetic subgroup, are upregulated upon bacterial infection. In contrast, IAN9 seems to form a specific subgroup and shows a unique downregulation upon bacterial infection. Infection with a virulent bacterial pathogen such as DC3000 triggers changes in plant cells caused by bacterial virulence activities (e.g., alteration of plant processes by T3E) and basal immune responses. Therefore, formally, transcriptional changes upon infection could be associated with bacterial virulence or plant immunity. In this case, the downregulation of IAN9 expression upon bacterial infection seems associated with the activation of immunity, because we detected similar expression patterns upon treatment with elicitors of immunity; namely, flg22 and SA. Interestingly, we detected a reduction in the amount of IAN9 transcripts after bacterial infection even in transgenic lines where IAN9 overexpression is driven by a constitutive $35 \mathrm{~S}$ promoter, suggesting a posttranscriptional regulation of IAN9 transcript abundance. Although the perception of flg22 leads to the production of SA
(Tsuda et al. 2008), our data show that the flg22-triggered reduction of IAN9 transcription takes place in sid2/NahG plants, indicating that this transcriptional change is independent of the observed SA-triggered reduction of IAN9 transcription. SAdeficient mutants are partially affected in flg22-triggered induction of defense-related genes, probably caused by lower levels of the flg22 receptor FLS2 (Yi et al. 2014). Our results indicate that these lower levels of FLS2 in sid2/NahG plants are nevertheless sufficient to trigger the IAN9 transcriptional repression, suggesting that IAN9 transcription may be highly sensitive to external biotic stimuli.

In addition to showing different expression patterns, the subcellular localization of IAN9 (mostly at the PM) is also different from that of IAN8 (mostly in the cytoplasm). This differential localization further suggests distinct functions for these two IAN family members. The C-terminal domain of IAN9, required for PM localization, is not present in other IAN proteins. This could indicate that the IAN9 mechanism for PM attachment is exclusive within the IAN family, although other IAN proteins could localize at the PM through different means.

Mutation of IAN9 causes increased resistance to DC3000, and this phenotype is rescued in complementation lines. It is worth considering the possibility that IAN9 is guarded by NLR, as has been shown for some regulators of immunity (Khan et al. 2016), therefore explaining the increased resistance in the ian 9 knockout mutant. However, the absence of developmental phenotypes or constitutive immune responses makes this possibility unlikely. Additionally, overexpression lines show a tendency for increased susceptibility, although this phenotype was not always significant or reproducible, and this trend was not observed upon inoculation with a hypovirulent DC3000 derivative strain. The lack of robustness in this phenotype could suggest that IAN9 transcript abundance is not rate limiting; however, it could also be due to the potential posttranscriptional regulation of IAN9 transcript abundance that we have seen in the IAN9 overexpression lines, which show a reduction in the amount of IAN9 transcripts after bacterial infection. The transient nature of IAN9 transcriptional repression upon SA treatment, returning to basal levels after $6 \mathrm{~h}$, may indicate that the negative regulation exerted by IAN9 could be required to

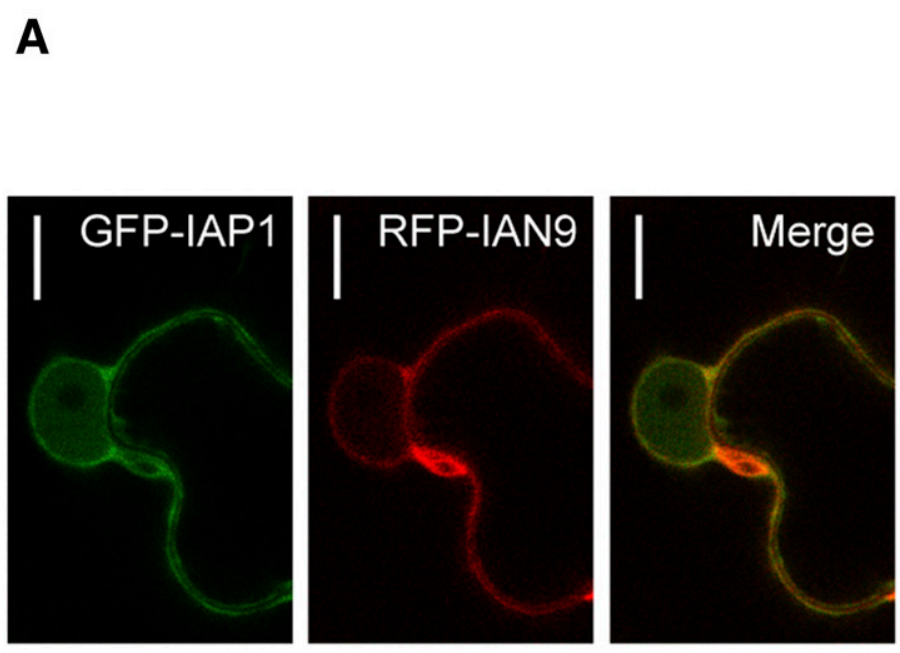

B

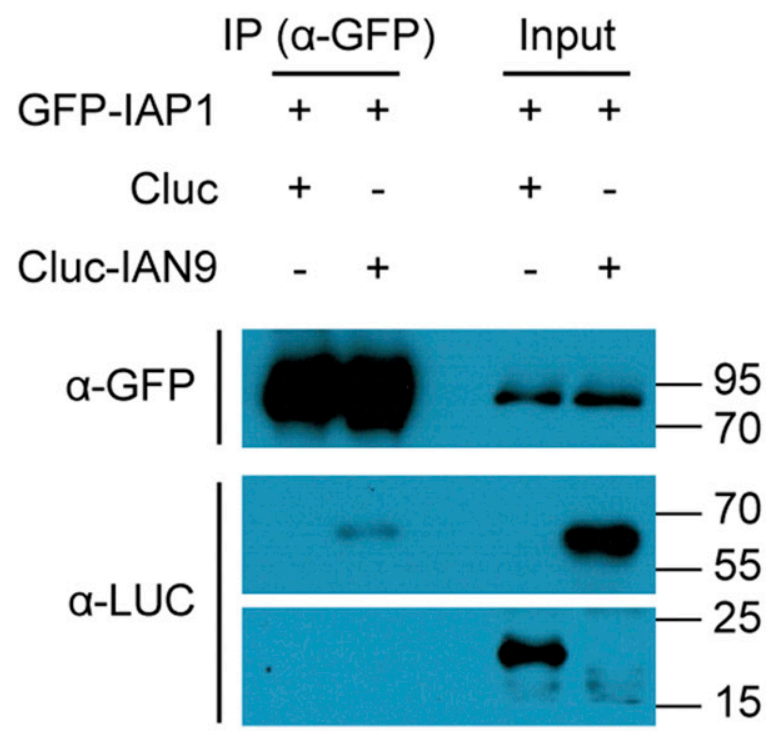

Fig. 5. Immune-associated nucleotide-binding (IAN)9-associated protein 1 (IAP1) interacts with IAN9 in Nicotiana benthamiana leaves. A, Confocal microscopy images showing the colocalization of green fluorescent protein (GFP)-IAP1 and red fluorescent protein (RFP)-IAN9 in N. benthamiana leaves. $\mathrm{Bar}=10 \mu \mathrm{m}$. B, Cluc or Cluc-IAN9 was coexpressed with GFP-IAP1 in $N$. benthamiana before immunoprecipitation (IP) using GFP-trap beads. Immunoblots were analyzed using anti-luc or anti-GFP antibody. Molecular weight $(\mathrm{kDa})$ marker bands are indicated for reference. Experiments were repeated three times with similar results. 
contribute to the repression of immune responses after immunity has been established.

Using IP followed by LC-MS/MS, we found IAP1 as a protein associated with IAN9. IAP1 contains three TPR domains, a C3HC4-type RING-finger domain, and an LON domain. Upon transient expression, IAP1 colocalizes with IAN9 in $N$. benthamiana cells. Genetic analysis indicates that both IAN9 and IAP1 negatively regulate immunity against DC3000. Given their physical association, it is possible that both proteins belong to a protein complex involved in the negative regulation of basal immunity against bacterial pathogens. Although we confirmed the association between IAN9 and IAP1 using targeted IP, we failed to detect a direct physical interaction using luciferase complementation or FRET-FLIM assays. Specific limitations of interaction techniques may be hindering the detection of a direct interaction; however, these data may also indicate that the interaction between IAN9 and IAP1 is indirect, perhaps mediated by other scaffolding components in the same protein complex. The Arabidopsis Interactome Database has reported several interactors for IAP1 in Y2H assays (Supplementary Table S2). Interestingly, several of these interacting proteins are transcription factors, and others have predicted nuclear localization or undergo nucleocytoplasmic relocalization associated with their activity. Considering that IAP1 interacts with several proteins with nuclear activities, it is tempting to speculate that the IAN9/IAP1 complex could associate with transcriptional regulators in the absence of biotic stress, acting as negative regulators of immune responses, and the complex may dissociate upon pathogen infection to allow for the activation of executor immune responses that restrict pathogen proliferation. This hypothetical model is in agreement with the observation that IAN9 does not seem to regulate early elicitor-induced responses (namely, flg22triggered ROS burst and MAPK activation). Given that ian 9 or iapl mutants do not show autoimmunity phenotypes, the final activation of immune responses may require additional activation by other immune regulators, of which the activity could be facilitated by the absence of IAN9 or IAP1.

The identification of sustainable sources of resistance against plant pathogens is essential to minimize crop losses due to diseases. A well-established approach consists on the mutation of negative regulators of immunity, or the so-called susceptibility genes, although this often leads to fitness costs or autoimmunity phenotypes, which obstruct their applicability in agriculture. Disease resistance based on loss-of-function mutations in mildew resistance locus o ( $m l o$ ) genes is one of the best-known examples of this approach (Kusch and Panstruga 2017). However, mlo mutations sometimes have pleiotropic effects that may affect plant yield and increase susceptibility to other pathogens (Kusch and Panstruga 2017). Altogether, our results reveal IAN9 and IAPI as suitable targets for biotechnological approaches to generate crops with increased disease resistance to bacterial pathogens, because both IAN9 and IAPI have orthologs in agriculturally important crop species (Supplementary Figs. S20 and S21). Interestingly, IAN9 and IAP1 behave as negative regulators of basal immunity, because ian9 and iap1 knockout plants show increased resistance to bacterial infection but they do not show obvious differences compared with wildtype plants in terms of size or development. It remains to be determined whether the mutation of IAN9 or IAPI orthologs in other plant species will have an impact on the plant response to other biotic or abiotic stresses. Alternative strategies to engineer resistance to plant diseases consider the pathogen-induced transcriptional and translational control of immune regulators (Gurr and Rushton 2005; Xu et al. 2017). These strategies show that it is possible to generate plants where the expression of defenserelated genes is restricted to cells undergoing pathogen attack, thus avoiding side effects on plant fitness. Current regulations hinder the use of transgenic plants to generate disease-resistant crops. Our IAN9 mutagenesis approach shows that it is feasible to design CRISPR/Cas9-mediated strategies to obtain stable nontransgenic mutant plants with increased resistance to pathogens and no obvious developmental defects, paving the way to a potential application in breeding for disease resistance.

\section{MATERIALS AND METHODS}

Plant materials and growth conditions.

Arabidopsis seed were sterilized with bleach solution $(20 \%$ bleach and $0.1 \%$ Triton X-100) for 2 to 3 min, then washed with sterile water four to five times and sown on solid 1/2 MS medium (2.21 g of Murashige \& Skoog basal medium with vitamins, $15 \mathrm{~g}$ of sucrose, and $7 \mathrm{~g}$ of agar per 1 liter). After stratification at $4^{\circ} \mathrm{C}$ for 3 days, the plates were transferred to a growth chamber $\left(22^{\circ} \mathrm{C}, 16 \mathrm{~h}\right.$ of light, and $8 \mathrm{~h}$ of darkness) for germination and growth. For experiments involving mature Arabidopsis plants, sterile seed were firstly stratified at $4^{\circ} \mathrm{C}$ for 3 days, then transferred to soil. Plants for bacterial infection assays were cultivated in a short-day chamber $\left(22^{\circ} \mathrm{C}\right.$, photoperiod of $8 \mathrm{~h}$ of light and $16 \mathrm{~h}$ of darkness, and $65 \%$ humidity). Plants for Agrobacterium transformation were grown in a long-day growth room $\left(22^{\circ} \mathrm{C}\right.$, photoperiod of $16 \mathrm{~h}$ of light and $8 \mathrm{~h}$ of darkness).

\section{Bacterial infections.}

P. syringae pv. tomato DC3000 and DC3000 (AvrRpt2) were streaked on selective 1/2 salt Luria-Bertani (LB) plates (10 g of
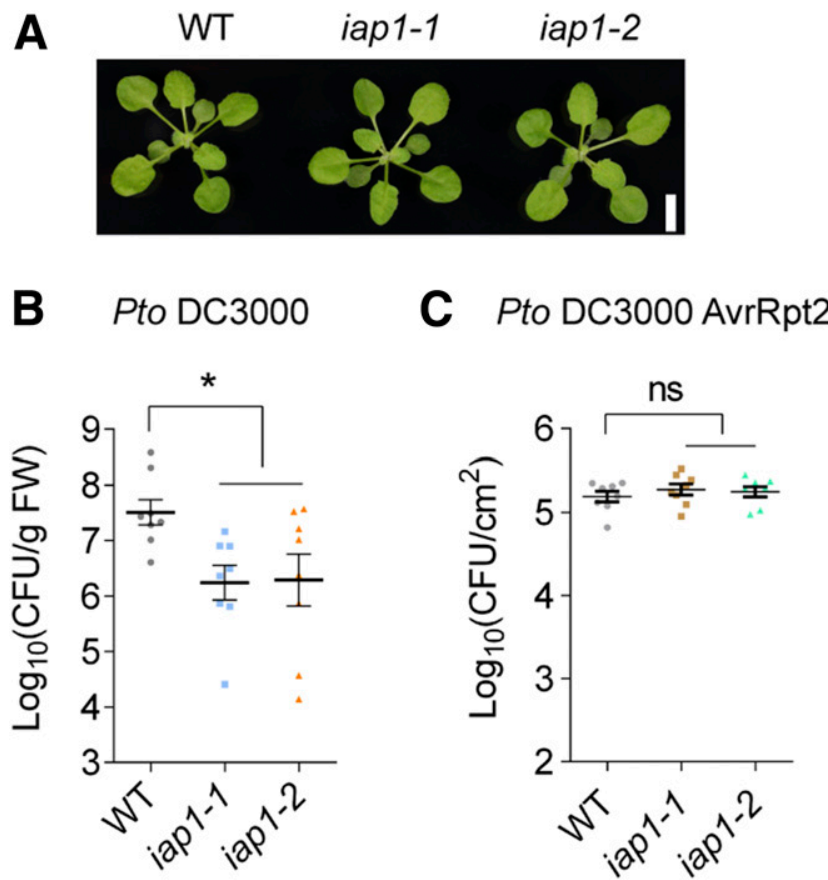

Fig. 6. Immune-associated nucleotide-binding (IAN)9-associated protein 1 (IAP1) negatively regulates plant immunity against $P$ seudomonas syringae pv. tomato DC3000. A, Photography of 4-week-old Col-0 wild type (WT), iap1-1, and iap1-2 plants, grown in a photoperiod of $8 \mathrm{~h}$ of light and $16 \mathrm{~h}$ of darkness. Scale bar is $0.5 \mathrm{~cm}$. B, Growth of surface (spray)-inoculated DC3000 (optical density at $600 \mathrm{~nm}\left[\mathrm{OD}_{600}\right]=0.1$ ) in wild-type (WT) Col-0, iap 1-1, and iap 1-2 mutant plants, 3 days postinoculation (dpi). Experiments repeated more than three times with similar results. C, Growth of DC3000 $\left(\right.$ AvrRpt2) $\left(\mathrm{OD}_{600}=0.001\right)$ infiltrated with a needleless syringe into wildtype (WT) Col-0, iap1-1, and iap1-2 mutant plants, 3 dpi. Experiments performed twice with similar results. Data were represented as means \pm standard error ( $n=8$ independent plants) (B and C). Statistical differences were calculated using a Student's $t$ test; "ns" indicates no significant difference and an asterisk indicates significant difference $(P<0.05)$. 
tryptone, $5 \mathrm{~g}$ of yeast extract, $5 \mathrm{~g}$ of $\mathrm{NaCl}$, and $15 \mathrm{~g}$ of agar per 1 liter) and cultivated at $22^{\circ} \mathrm{C}$ for 2 days. For DC 3000 , bacteria were collected from the plates into sterile water, the optical density (OD) was adjusted to a value of $0.1\left(5 \times 10^{7} \mathrm{CFU} / \mathrm{ml}\right)$ or $0.2\left(10^{8} \mathrm{CFU} / \mathrm{ml}\right)$, and silwet-L77 was added to a final concentration of $0.03 \%$ before performing spray inoculation onto 3- to 4-week-old soil-grown Arabidopsis under short-day conditions. The plants were then covered with cling wrap for $3 \mathrm{~h}$. The whole plants were harvested in $1.5-\mathrm{ml}$ microcentrifuge tubes and weighed. For DC3000 (AvrRpt2), the OD was adjusted to $0.1\left(5 \times 10^{7} \mathrm{CFU} / \mathrm{ml}\right)$ or $0.001\left(10^{5} \mathrm{CFU} / \mathrm{ml}\right)$. The bacterial suspension was pressure infiltrated into 5- to 6-weekold short-day-grown Arabidopsis leaves with a needleless syringe (3 leaves/plant), and leaf discs were collected into $1.5-\mathrm{ml}$ tubes using a leaf punch at 3 days postinoculation. In both cases, plant tissues were ground and homogenized in sterile water before plating serial dilutions on selective $1 / 2$ salt LB agar plates. The plates were placed at $28^{\circ} \mathrm{C}$ for 1.5 days and the bacterial growth was calculated as CFU.

For gene expression assays, sterile seed were sown on $1 / 2$ solid MS medium to germinate and grown for 3 to 4 days in long-day conditions. Seedlings were then transferred into $1 / 2$ liquid MS medium in 12-well culture plates (Thermo Fisher Scientific, Waltham, MA, U.S.A.), and grown for another 6 or 7 days. Each well contained three seedlings (pulled together as one sample), and every experiment included three independent samples from three independent wells. Seedlings were inoculated with DC3000 or DC3000 (AvrRpt2) at $5 \times 10^{7}$ $\mathrm{CFU} / \mathrm{ml}$, and samples were collected $6 \mathrm{~h}$ after inoculation.

\section{Treatments with immune elicitors.}

Sterile seed were sown on $1 / 2$ solid MS medium to germinate and grown for 3 to 4 days in long-day conditions. Seedlings were then transferred into $1 / 2$ liquid MS medium in 12-well culture plates (Thermo Fisher Scientific), and grown for another 6 or 7 days. Each well contained three seedlings (pulled together as one sample), and every experiment included three independent samples from three independent wells. The flg22 peptide or SA were added into the liquid medium to a final concentration of $100 \mathrm{nM}$ and $0.5 \mathrm{mM}$, respectively. All of the plates were incubated on a shaker for 5 to $10 \mathrm{~min}$ following addition of the elicitor. Samples were harvested into $1.5-\mathrm{ml}$ tubes at different time points after treatment, as indicated in the figures.

\section{Root growth assay.}

Sterile seed were sown on $1 / 2$ solid MS medium and stratified at $4^{\circ} \mathrm{C}$ for 3 days. Then, the plates were placed into a growth chamber for 1 day. The germinated seedlings were transferred to the new 1/2 solid MS medium (square plates). Square plates were placed vertically into a growth chamber and root length was measured 5 days later.

\section{RNA isolation, RT-PCR, and RT-qPCR.}

For RNA extraction, plant tissues were collected in $1.5-\mathrm{ml}$ microfuge tubes with one metal bead and the tubes were immediately placed into liquid nitrogen. Samples were ground thoroughly with a TissueLyser (QIAGEN, Duesseldorf, Nordrhein-Westfalen, Germany) for 1 min and placed back in liquid nitrogen. Total RNA was extracted with the E.Z.N.A. Plant RNA kit (Omega Bio-Tek, Norcross, GA, U.S.A.) with incolumn DNA digestion and an additional sample treatment with DNAase (Omega Bio-Tek). First-strand cDNA was synthesized using the iScript cDNA synthesis kit (Bio-Rad, Hercules, CA, U.S.A.) with a volume of $20 \mu$ l. For RT-PCR, the PCR was performed in $50 \mu \mathrm{l}$ using the Q5 Hot Start High-Fidelity DNA polymerase (New England Biolabs, Ipswich, MA, U.S.A.) with
35 or 38 cycles. For RT-qPCR, PCR were performed in $20 \mu 1$ using the iTaq Universal SYBR Green Supermix kit (Bio-Rad) in the StepOnePlus Real-Time PCR System (Applied Biosystems, Foster City, CA, U.S.A.). Data were analyzed with Excel and GraphPad Prism 6.

\section{Confocal microscopy imaging.}

Cotyledons from 3- to 4-day-old long-day-grown Arabidopsis seedlings were imaged using a Confocal Laser Scanning Microscope Platform: Leica TCS SP8 (Leica, Mannheim, Germany). The GFP was excited with an argon laser at $488 \mathrm{~nm}$, and its emission was detected at 500 to $550 \mathrm{~nm}$. For plasmolysis assays, cotyledons were placed into a $1 \mathrm{M} \mathrm{NaCl}$ solution on glass slides, and GFP was observed and recorded after 5 to 10 min. For FM4-64 staining, cotyledons from 4-day-old seedlings were cut and soaked into FM4-64 solution at $5 \mathrm{ng} / \mu \mathrm{l}$ as described previously (Beck et al. 2012) for $5 \mathrm{~min}$; samples were then transferred to water on glass slides and covered with coverslips. For dual-channel simultaneous observation, the fluorescence signal of FM4-64 was excited with an argon laser at $561 \mathrm{~nm}$ and its emission was observed at 580 to $650 \mathrm{~nm}$; the GFP was excited at $488 \mathrm{~nm}$ and observed at 500 to $550 \mathrm{~nm}$. For colocalization assays, 3-week-old $N$. benthamiana leaves were coinfiltrated with Agrobacterium GV3101 (pMP90) carrying plasmids to express RFP-IAN9 and GFP-IAPl. Leaves were coinfiltrated with the GV3101 strain carrying plasmids to express RFP-IAN9 and GFP as control. Leaf discs $(3 \mathrm{~mm}$ in diameter) were punched from the whole leaf $24 \mathrm{~h}$ postinfiltration and transferred to water on glass slides. For dualchannel image acquisition, GFP and RFP were excited at 488 and $561 \mathrm{~nm}$ respectively; emission was collected at 500 to $550 \mathrm{~nm}$ for GFP and 580 to $650 \mathrm{~nm}$ for RFP.

\section{MAPK activation and Western blot assays.}

For protein extraction, Arabidopsis seedlings or leaf discs from $N$. benthamiana were ground with a Tissue Lyser (QIAGEN, Hilden, Nordrhein-Westfalen, Germany). Samples were then resuspended in lysis buffer $(2 \times$ loading buffer: $100 \mathrm{mM}$ Tris$\mathrm{HCl}$ [pH 6.8], 10\% Glycerol, 2\% sodium dodecyl sulfate [SDS], and $0.03 \%$ bromophenol blue), boiled at $95^{\circ} \mathrm{C}$ for $10 \mathrm{~min}$, and centrifuged at $14,000 \times g$ for 5 min before loading in SDS polyacrylamide gel electrophoresis (PAGE) acrylamide gels. Western blots were performed using anti-GFP (Sigma G6795), antiluciferase (Sigma L0159), anti-mouse immunoglobulin G (IgG)peroxidase (Sigma A2554), and anti-rabbit IgG-peroxidase (Sigma A0545).

MAPK activation assays were performed as previously described (Macho et al. 2012), with minor modifications. Briefly, 7-day-old Arabidopsis seedlings grown on solid 1/2 MS medium were transferred to water and then treated with $100 \mathrm{nM}$ flg 22 for $10 \mathrm{~min}$ after vacuuming for $5 \mathrm{~min}$ ( $15 \mathrm{~min}$ in total). AntipMAPK (Phospho-p44/42 MAPK [Erk1/2] [Thr202/Tyr204] XP Rabbit mAb; Cell Signaling 4370) was dissolved in 3\% gelatin (Sigma G7041) and used to hybridize the membranes. All membranes were stained with Ponceau stain (Sangon Biotech, Shanghai, China) to verify equal loading.

\section{ROS burst.}

ROS burst assays were conducted as previously described (Sang and Macho 2017). Briefly, 4-mm leaf discs from 5- to 6week-old Arabidopsis plants grown in short-day conditions were transferred to 96-well microplates (PerkinElmer, Waltham, MA, U.S.A.) with $100 \mu$ l of Mili-Q water per well and incubated overnight. Water was then removed and ROS burst was elicited by adding $100 \mu \mathrm{l}$ of a solution containing $100 \mathrm{ng}$ flg22, $100 \mu \mathrm{M}$ luminol, and $20 \mu \mathrm{g} / \mathrm{ml}$ horseradish peroxidase. 
The luminescence was recorded over 40 min using a Thermo Scientific VARIOSKAN FLASH (Thermo Fisher Scientific).

\section{Co-IP and large-scale IP for LC-MS/MS.}

Leaves from 3- to 4-week-old $N$. benthamiana plants were coinfiltrated with Agrobacterium GV3101 (pMP90) carrying plasmids to express GFP-IAPl and Cluc-IAN9; leaves coinfiltrated with GV3101 carrying plasmids to express GFP-AIPl and $C l u c$ or GFP and Cluc were used as controls. Total proteins were extracted $24 \mathrm{~h}$ later and IP was performed with GFP-trap beads (Chromotek, Am Klopferspitz, Planegg-Martinsried, Germany), as described previously (Sang et al. 2018). Proteins were stripped from the beads by boiling in $50 \mu \mathrm{l}$ of SDS loading buffer for $10 \mathrm{~min}$. Immunoprecipitated proteins were separated on SDSPAGE acrylamide gels and Western blots were performed as described above. Large-scale IP assays for LC-MS/MS were performed as described before (Kadota et al. 2016; Sang et al. 2018), using $5 \mathrm{~g}$ of 10-day-old Arabidopsis seedlings before or after treatment with $100 \mathrm{nM}$ flg22 or $0.5 \mathrm{mM} \mathrm{SA}$.

\section{Plasmid construction.}

The sequence of all of the primes used in this study is shown in Supplementary Table S3. A free GFP fragment (with stop codon) was amplified from the plasmid pGWB505 (Nakagawa et al. 2007). The purified PCR product was transferred into entry vector $\mathrm{pDONR/ZEO} \mathrm{(Thermo} \mathrm{Fisher} \mathrm{Scientific)} \mathrm{by} \mathrm{BP}$ reaction, then recombined into pGWB602 (35S promoter, no tag) (Nakagawa et al. 2007) using LR reaction to yield the pGWB602-GFP plasmid. The coding DNA sequence of IAN9, IAN9 $(\triangle C 27 a a), I A N 8$, and IAPI (all with stop codon) were amplified from cDNA of whole Arabidopsis seedlings using primers containing attB1attB2 sites. The PCR fragments were ligated into $\mathrm{pDONR} / \mathrm{ZEO}$ by $\mathrm{BP}$ reaction, then recombined with the binary vector pGWB606 (35S pro, N-sGFP) (Nakagawa et al. 2007) to yield the plasmids pGWB606-IAN9, pGWB606IAN9 $(\triangle C 27 a a)$, pGWB606-IAN8, and pGWB606-IAP1 through an LR reaction. pDONR/ZEO-IAN9 was also recombined with the binary vector pGWB555 (35S promoter, N-mRFP) to yield the plasmid pGWB555-IAN9 through an LR reaction.

For luciferase complementation assays, the original vectors (Chen et al. 2008) were modified to make them Gateway compatible. The binary vector pCAMBIA1300-nLUC was digested with SacI and SalI and pCAMBIA1300-cLUC was digested with KpnI and SalI. The gateway cassette was then amplified from pGWB505 with primers Nluc-F/R and Cluc-F/R and PCR products were cloned into the digested pCAMBIA1300 vectors using ClonExpress II One Step Cloning Kit (Vazyme Biotech, Nanjing, China) to yield the new version of the binary plasmids pGWBNluc and pGWB-Cluc containing the Gateway cassette. Then, pDONR/ZEO-IAPl (without stop codon) and pDONR/ZEO-IAN9 were separately recombined with pGWB-Nluc and pGWB-Cluc through an LR reaction to yield the destination vectors pGWBC3HC4-Nluc and pGWB-Cluc-IAN9.

For CRISPR/Cas9-mediated mutagenesis, the detailed sites were predicted using the CRISPR Design tool. PCR products were ligated into the pCAS9 plasmid (Feng et al. 2013) using T4 DNA ligase (New England Biolabs).

\section{Arabidopsis transformation.}

For the generation of Arabidopsis transgenic lines, Agrobacterium-mediated transformation was performed according to the method described before (Clough and Bent 1998). The Agrobacterium tumefaciens strain GV3101 (pMP90) carrying the desired plasmids (pGWB602-GFP, pGWB605-IAN9, pGWB606-IAN9, pGWB605-IAN8, and pGWB606-IAN9 [ $\triangle \mathrm{C}-27 \mathrm{aa}])$ were cultured overnight at $28^{\circ} \mathrm{C}$, then spun down and resuspended to an OD at $600 \mathrm{~nm}\left(\mathrm{OD}_{600}\right)=1$ in $50 \mathrm{ml}$ of
$5 \%$ sucrose and $0.03 \%$ silwet-L77 solution. The fertilized siliques from 5- to 6-week-old Col-0 wild-type plants (grown in a photoperiod of $16 \mathrm{~h}$ of light and $8 \mathrm{~h}$ of darkness) were removed before flower dipping. After dipping for 1 to $2 \mathrm{~min}$, the plants were covered with polyethylene cling wrap for 16 to $24 \mathrm{~h}$ in the dark, then put back to normal growth conditions until seed collection. Finally, homozygous transgenic lines were obtained after resistance selection (BASTA at $15 \mu \mathrm{g} / \mathrm{ml}$ and hygromycin $\mathrm{B}$ at $25 \mu \mathrm{g} / \mathrm{ml}$ ) and segregation ratio calculation.

For the generation of the ian9 mutant, A. tumefaciens GV3101 carrying the plasmid pCas9 (35S, AtU6, Hpt)-IAN9 was used to transform Col-0 wild-type Arabidopsis plants. T1 plants were selected in $1 / 2$ solid MS (hygromycin B at $25 \mu \mathrm{g} / \mathrm{ml}$ ), then transferred to soil. Genomic DNA was extracted from 2week-old soil-grown T1 plants using the cetyltrimethylammonium bromide method (Doyle and Doyle 1987). IAN9 was amplified from independent DNA samples and sequenced. The plants that possessed a double peak in the sequencing results of the target sequence were chosen for collection of T1 seed. Independent T1 seed were sown on selective (hygromycin B at $25 \mu \mathrm{g} / \mathrm{ml}$ ) and nonselective $1 / 2$ solid MS medium and the segregation ratio was calculated. Lines with 3:1 ratio were transferred to the soil. IAN9 and $C A S 9$ genes were amplified by PCR, and plants without the CAS9 product were selected. Homozygous CRISPR/CAS9 mutant ian9 lines without CAS9 background were selected for further experiments.

\section{Agrobacterium-mediated transient expression in $N$. benthamiana.}

A. tumefaciens GV3101 carrying the desired plasmid (pGWB606-IAP1, pGWB555-IAN9, pGWB-Cluc, pGWBCluc-IAN9, or pGWB-Nluc-IAP1) were grown on selective LB plates (10 $\mathrm{g}$ of tryptone, $5 \mathrm{~g}$ of yeast extract, $10 \mathrm{~g}$ of $\mathrm{NaCl}$, and $15 \mathrm{~g}$ of agar per liter) and cultivated at $28^{\circ} \mathrm{C}$ for 2 days. Before infiltrating 3- to 4-week-old N. benthamiana plants, Agrobacterium cells were resuspended in the infiltration buffer (10 $\mathrm{mM} \mathrm{MgCl}_{2}, 10 \mathrm{mM}$ morpholineethanesulfonic acid $[\mathrm{pH}$ 5.6], and $150 \mu \mathrm{M}$ acetosyringone) directly from plates, and diluted to an $\mathrm{OD}_{600}$ of 0.5 or 1 , depending on the expression and stability of the different proteins. Samples were collected $24 \mathrm{~h}$ after Agrobacterium infiltration.

\section{Luciferase complementation assay.}

Luciferase complementation assays were performed as previously described (Chen et al. 2008). Three-week-old $N$. benthamiana leaves were coinfiltrated with Agrobacterium GV3101 (pMP90) carrying the plasmids to induce the expression of IAPI-Nluc and Cluc-IAN9. One day after inoculation, the whole leaves were cut and sprayed with luciferin solution (1 mM luciferin [Sigma] and 0.02\% Triton X-100]. The fluorescence signal was recorded using a Lumazone 1300B (Scientific Instrument, West Palm Beach, FL, U.S.A.) for $10 \mathrm{~min}$ after $5 \mathrm{~min}$ in the dark.

\section{FRET-FLIM.}

Three-week-old $N$. benthamiana leaves were coinfiltrated with Agrobacterium GV3101 (pMP90) carrying the plasmids to induce the expression of GFP-IAPl with RFP-IAN9 (FRET pair: donor + acceptor); leaves infiltrated with GV3101 inducing the expression of GFP-IAP1 with RFP (donor + free RFP) and GFP-IAPI (donor alone) were used as negative control. FRET-FLIM experiments were performed on a Leica TCS SMD FLCS confocal microscope. Leaf discs $(6 \mathrm{~mm}$ in diameter) of $N$. benthamiana plants transiently coexpressing donor and acceptor were visualized 1 day after agroinfiltration. The lifetime of the donor $(\tau)$ was collected and analyzed as described by Rosas-Diaz et al. (2018). 


\section{Chemicals.}

The flg22 peptide (TRLSSGLKINSAKDDAAGLQIA) was purchased from Abclonal, USA. Sequencing-grade modified trypsin was purchased from Promega Corp. (Madison, WI, U.S.A.). All other chemicals were purchased from SigmaAldrich (St. Louis), unless otherwise stated.

\section{ACKNOWLEDGMENTS}

We thank members of the Macho and Lozano-Durán laboratories for helpful discussions; J. Li, J.-M. Zhou, and J.-K. Zhu for sharing biological materials; X. Jian for technical and administrative assistance during this work; the PSC Cell Biology core facility for assistance with confocal microscopy; and the PSC Proteomics core facility for LC-MS/MS analysis.

\section{LITERATURE CITED}

Beck, M., Zhou, J., Faulkner, C., MacLean, D., and Robatzek, S. 2012. Spatio-temporal cellular dynamics of the Arabidopsis flagellin receptor reveal activation status-dependent endosomal sorting. Plant Cell 24: 4205-4219.

Bigeard, J., Colcombet, J., and Hirt, H. 2015. Signaling mechanisms in pattern-triggered immunity (PTI). Mol. Plant 8:521-539.

Boller, T., and Felix, G. 2009. A renaissance of elicitors: Perception of microbe-associated molecular patterns and danger signals by patternrecognition receptors. Annu. Rev. Plant Biol. 60:379-406.

Bolte, S., Talbot, C., Boutte, Y., Catrice, O., Read, N. D., and SatiatJeunemaitre, B. 2004. FM-dyes as experimental probes for dissecting vesicle trafficking in living plant cells. J. Microsc. 214:159-173.

Bourne, H. R., Sanders, D. A., and McCormick, F. 1991. The GTPase superfamily: Conserved structure and molecular mechanism. Nature 349:117-127.

Campos, M. L., Yoshida, Y., Major, I. T., de Oliveira Ferreira, D., Weraduwage, S. M., Froehlich, J. E., Johnson, B. F., Kramer, D. M., Jander, G., Sharkey, T. D., and Howe, G. A. 2016. Rewiring of jasmonate and phytochrome B signalling uncouples plant growth-defense tradeoffs. Nat. Commun. 7:Article 12570.

Chen, H., Zou, Y., Shang, Y., Lin, H., Wang, Y., Cai, R., Tang, X., and Zhou, J. M. 2008. Firefly luciferase complementation imaging assay for protein-protein interactions in plants. Plant Physiol. 146:368-376.

Chiang, Y.-H., and Coaker, G. 2015. Effector triggered immunity: NLR immune perception and downstream defense responses. Arabidopsis Book 13:e0183.

Clough, S. J., and Bent, A. F. 1998. Floral dip: A simplified method for Agrobacterium-mediated transformation of Arabidopsis thaliana. Plant J. 16:735-743.

Cook, D. E., Mesarich, C. H., and Thomma, B. P. H. J. 2015. Understanding plant immunity as a surveillance system to detect invasion. Annu. Rev. Phytopathol. 53:541-563.

Couto, D., and Zipfel, C. 2016. Regulation of pattern recognition receptor signalling in plants. Nat. Rev. Immunol. 16:537-552.

Delaney, T. P., Uknes, S., Vernooij, B., Friedrich, L., Weymann, K., Negrotto, D., Gaffney, T., Gut-Rella, M., Kessmann, H., Ward, E., and Ryals, J. 1994. A central role of salicylic acid in plant disease resistance. Science 266:1247-1250.

de Wit, M., Spoel, S. H., Sanchez-Perez, G. F., Gommers, C. M. M., Pieterse, C. M. J., Voesenek, L. A. C. J., and Pierik, R. 2013. Perception of low red:far-red ratio compromises both salicylic acidand jasmonic acid-dependent pathogen defences in Arabidopsis. Plant J. 75:90-103.

Doyle, J. J., and Doyle, J. L. 1987. A rapid DNA isolation procedure for small quantities of fresh leaf tissue. Phytochem. Bull. 19:11-15.

Feng, Z., Zhang, B., Ding, W., Liu, X., Yang, D. L., Wei, P., Cao, F., Zhu, S., Zhang, F., Mao, Y., and Zhu, J. K. 2013. Efficient genome editing in plants using a CRISPR/Cas system. Cell Res. 23:1229-1232.

Fischer-Parton, S., Parton, R. M., Hickey, P. C., Dijksterhuis, J., Atkinson, H. A., and Read, N. D. 2000. Confocal microscopy of FM4-64 as a tool for analysing endocytosis and vesicle trafficking in living fungal hyphae. J. Microsc. 198:246-259.

Gurr, S. J., and Rushton, P. J. 2005. Engineering plants with increased disease resistance: How are we going to express it? Trends Biotechnol. 23:283-290.

Huot, B., Yao, J., Montgomery, B. L., and He, S. Y. 2014. Growth-defense tradeoffs in plants: A balancing act to optimize fitness. Mol. Plant 7: 1267-1287.
Kadota, Y., Macho, A. P., and Zipfel, C. 2016. Immunoprecipitation of plasma membrane receptor-like kinases for identification of phosphorylation sites and associated proteins. Methods Mol. Biol. 1363: 133-144.

Khan, M., Subramaniam, R., and Desveaux, D. 2016. Of guards, decoys, baits and traps: Pathogen perception in plants by type III effector sensors. Curr. Opin. Microbiol. 29:49-55.

Kusch, S., and Panstruga, R. 2017. mlo-Based resistance: An apparently universal "weapon" to defeat powdery mildew disease. Mol. PlantMicrobe Interact. 30:179-189.

Liu, C., Wang, T., Zhang, W., and Li, X. 2008. Computational identification and analysis of immune-associated nucleotide gene family in Arabidopsis thaliana. J. Plant Physiol. 165:777-787.

Macho, A. P. 2016. Subversion of plant cellular functions by bacterial typeIII effectors: Beyond suppression of immunity. New Phytol. 210:51-57.

Macho, A. P., Boutrot, F., Rathjen, J. P., and Zipfel, C. 2012. Aspartate oxidase plays an important role in Arabidopsis stomatal immunity. Plant Physiol. 159:1845-1856.

Macho, A. P., and Zipfel, C. 2014. Plant PRRs and the activation of innate immune signaling. Mol. Cell 54:263-272.

Macho, A. P., and Zipfel, C. 2015. Targeting of plant pattern recognition receptor-triggered immunity by bacterial type-III secretion system effectors. Curr. Opin. Microbiol. 23:14-22.

Mao, Y., Zhang, H., Xu, N., Zhang, B., Gou, F., and Zhu, J. K. 2013. Application of the CRISPR-Cas system for efficient genome engineering in plants. Mol. Plant 6:2008-2011.

Melotto, M., Underwood, W., Koczan, J., Nomura, K., and He, S. Y. 2006. Plant stomata function in innate immunity against bacterial invasion. Cell 126:969-980.

Nakagawa, T., Kurose, T., Hino, T., Tanaka, K., Kawamukai, M., Niwa, Y., Toyooka, K., Matsuoka, K., Jinbo, T., and Kimura, T. 2007. Development of series of gateway binary vectors, pGWBs, for realizing efficient construction of fusion genes for plant transformation. J. Biosci. Bioeng. 104:34-41.

Nitta, T., Nasreen, M., Seike, T., Goji, A., Ohigashi, I., Miyazaki, T., Ohta, T., Kanno, M., and Takahama, Y. 2006. IAN family critically regulates survival and development of T lymphocytes. PLoS Biol. 4:e103.

Nitta, T., and Takahama, Y. 2007. The lymphocyte guard-IANs: Regulation of lymphocyte survival by IAN/GIMAP family proteins. Trends Immunol. 28:58-65.

Reuber, T. L., and Ausubel, F. M. 1996. Isolation of Arabidopsis genes that differentiate between resistance responses mediated by the RPS2 and RPM1 disease resistance genes. Plant Cell 8:241-249.

Rosas-Diaz, T., Zhang, D., Fan, P., Wang, L., Ding, X., Jiang, Y., JimenezGongora, T., Medina-Puche, L., Zhao, X., Feng, Z., Zhang, G., Liu, X., Bejarano, E. R., Tan, L., Zhang, H., Zhu, J. K., Xing, W., Faulkner, C., Nagawa, S., and Lozano-Duran, R. 2018. A virus-targeted plant receptorlike kinase promotes cell-to-cell spread of RNAi. Proc. Natl. Acad. Sci. U.S.A. 115:1388-1393.

Sang, Y., and Macho, A. P. 2017. Analysis of PAMP-Triggered ROS Burst in Plant Immunity. Methods Mol. Biol. 1578:143-153.

Sang, Y., Wang, Y., Ni, H., Cazale, A. C., She, Y. M., Peeters, N., and Macho, A. P. 2018. The Ralstonia solanacearum type III effector RipAY targets plant redox regulators to suppress immune responses. Mol. Plant Pathol. 19:129-142.

Scheres, B., and van der Putten, W. H. 2017. The plant perceptron connects environment to development. Nature 543:337-345.

Stael, S., Kmiecik, P., Willems, P., Van Der Kelen, K., Coll, N. S., Teige, M., and Van Breusegem, F. 2015. Plant innate immunity-Sunny side up? Trends Plant Sci. 20:3-11.

Takai, Y., Sasaki, T., and Matozaki, T. 2001. Small GTP-binding proteins. Physiol. Rev. 81:153-208.

Tsuda, K., Glazebrook, J., and Katagiri, F. 2008. The interplay between MAMP and SA signaling. Plant Signal. Behav. 3:359-361.

Vernoud, V., Horton, A. C., Yang, Z., and Nielsen, E. 2003. Analysis of the small GTPase gene superfamily of Arabidopsis. Plant Physiol. 131: 1191-1208.

Vlot, A. C., Dempsey, D. A., and Klessig, D. F. 2009. Salicylic acid, a multifaceted hormone to combat disease. Annu. Rev. Phytopathol. 47:177-206.

Wang, Z. Y., Seto, H., Fujioka, S., Yoshida, S., and Chory, J. 2001. BRI1 is a critical component of a plasma-membrane receptor for plant steroids. Nature 410:380-383.

Weiss, Y., Forêt, S., Hayward, D. C., Ainsworth, T., King, R., Ball, E. E., and Miller, D. J. 2013. The acute transcriptional response of the coral Acropora millepora to immune challenge: Expression of GiMAP/IAN genes links the innate immune responses of corals with those of mammals and plants. BMC Genomics 14:400. 
Wildermuth, M. C., Dewdney, J., Wu, G., and Ausubel, F. M. 2001. Isochorismate synthase is required to synthesize salicylic acid for plant defence. Nature 414:562-565.

Win, J., Chaparro-Garcia, A., Belhaj, K., Saunders, D. G., Yoshida, K., Dong, S., Schornack, S., Zipfel, C., Robatzek, S., Hogenhout, S. A., and Kamoun, S. 2012. Effector biology of plant-associated organisms: Concepts and perspectives. Cold Spring Harb. Symp. Quant. Biol. 77: 235-247.

Xu, G., Yuan, M., Ai, C., Liu, L., Zhuang, E., Karapetyan, S., Wang, S., and Dong, X. 2017. uORF-mediated translation allows engineered plant disease resistance without fitness costs. Nature 545:491-494.
Yi, S. Y., Shirasu, K., Moon, J. S., Lee, S. G., and Kwon, S. Y. 2014. The activated SA and JA signaling pathways have an influence on flg22triggered oxidative burst and callose deposition. PLoS One 9:e88951.

Zipfel, C. 2014. Plant pattern-recognition receptors. Trends Immunol. 35 345-351.

\section{AUTHOR-RECOMMENDED INTERNET RESOURCES}

The Arabidopsis Interactome Database:

http://interactome.dfci.harvard.edu/A_thaliana/index.php

CRISPR Design tool: http://crispr.mit.edu/ 